\title{
Management of Hypertension in Intrapericardial Paraganglioma
}

\author{
Nicola Rotolo, ${ }^{1}$ Andrea Imperatori, ${ }^{1}$ Alessandro Bacuzzi, ${ }^{2}$ Valentina Conti, ${ }^{1}$ \\ Massimo Castiglioni, ${ }^{1}$ and Lorenzo Dominioni ${ }^{1}$ \\ ${ }^{1}$ Center for Thoracic Surgery, Department of Surgical and Morphological Sciences, University of Insubria, Via Guicciardini 9, \\ 21100 Varese, Italy \\ ${ }^{2}$ Department of Anaesthesiology, Ospedale di Circolo, Via Guicciardini 9, 21100 Varese, Italy
}

Correspondence should be addressed to Lorenzo Dominioni; lorenzo.dominioni@uninsubria.it

Received 29 November 2013; Accepted 7 January 2014; Published 13 February 2014

Academic Editor: B. Gil Extremera

Copyright (C) 2014 Nicola Rotolo et al. This is an open access article distributed under the Creative Commons Attribution License, which permits unrestricted use, distribution, and reproduction in any medium, provided the original work is properly cited.

\begin{abstract}
Functioning paraganglioma is extra-adrenal catecholamine-secreting tumours that may cause secondary hypertension. Primary intrapericardial paragangliomas are very rare and are located adjacent to the great vessels or heart, typically near the left atrium. These tumours are an exceptionally uncommon finding during the investigation of refractory hypertension. However, in recent years, intrapericardial paragangliomas have been diagnosed incidentally with increased frequency, due to the extensive use of radiologic chest imaging. The mainstay of treatment of functioning intrapericardial paraganglioma is surgical removal, which usually achieves blood pressure normalization. Due to the locations of these tumours, the surgical approach is through a median sternotomy or posterolateral thoracotomy, and manipulation-induced catecholamine release may cause paroxysmal hypertension. Typically in these patients, blood pressure fluctuates dramatically intra- and post-operatively, increasing the risk of cardiovascular complications. We review here the current modalities of perioperative fluid and hypotensive drug administration in the setting of surgery for functioning intrapericardial paraganglioma and discuss the recently proposed paradigm shift that omits preoperative preparation.
\end{abstract}

\section{Introduction}

Hypertension is predominantly essential, but in $15 \%$ of cases it is secondary to renal or endocrine diseases [1]. Pheochromocytoma and extra-adrenal paraganglioma are rare chromaffin tumours arising from neural crest tissue that develops into sympathetic and parasympathetic paraganglia throughout the body. These tumours may secrete large amounts of catecholamines and represent an uncommon identifiable cause of resistant hypertension [2]. The World Health Organization classification uses the term pheochromocytoma only for tumours of adrenal medulla origin and the term paraganglioma for similar lesions that arise from other locations [3].

Catecholamine-secreting tumours occur in less than $0.1 \%$ of the hypertensive population; the peak incidence is between the third and fifth life decades, with both genders being equally affected. Twenty-five percent of pheochromocytoma and paraganglioma occur in the setting of familial syndromes
(SDH gene mutations; MEN 2A/B; von Hipple-Lindau; Neurofibromatosis I; Carney triad). Thus, family history should be carefully investigated in patients with catecholaminesecreting tumours, and all relatives should be screened for hereditary syndromes [4-6]. Most paragangliomas are solitary, but they tend to be multicentric in hereditary syndromes; in an institutional series of 12 cases, $25 \%$ were multicentric [5].

\section{Incidence and Prognosis of Intrapericardial Paraganglioma}

Less than $2 \%$ of reported paraganglioma are located in the chest and intrapericardial paraganglioma (IP) is even rarer [5].

Most IPs are large, ranging in size from 3 to $8 \mathrm{~cm}[6,7]$; in the 72 cases listed in Table 1, the average tumour diameter 
TABLE 1: Published case reports of intrapericardial paraganglioma in 1994-2013.

\begin{tabular}{|c|c|c|c|c|c|c|c|c|}
\hline Authors & Year & No. of Pts & Symptoms & $\begin{array}{l}\text { Tumour } \\
\text { location }\end{array}$ & $\begin{array}{c}\text { Catechol. secretion } \\
\text { increase }\end{array}$ & Treatment & $\begin{array}{c}\text { Hypertension } \\
\text { after surgery }\end{array}$ & $\mathrm{CPBP}$ \\
\hline Gonzale-Lopez et al. [25] & 2013 & 1 & Hered. Synd. & Retroc. & Yes & Surg. & No & Yes \\
\hline Liu et al. [26] & 2013 & 1 & HT. & Interatrial. & Yes & Surg. & No & Yes \\
\hline Wang et al. [27] & 2013 & 1 & HT. & R.A. & n.s. & Surg. & No & Yes \\
\hline Tracy and Wein [28] & 2013 & 1 & Hered. Synd. & R.A. & Yes & n.s. & n.s. & n.s. \\
\hline Ramlawi et al. [9] & 2012 & 7 & $5 / 7$ Pts & 71\% L.A. & No & Surg. & No & Yes \\
\hline Marshall et al. [29] & 2012 & 1 & HT. & R.A. & Yes & Surg. & No & Yes \\
\hline Huo et al. [30] & 2012 & 2 & Palpitation & L.A.; R.A. & Yes & Surg. & No & Yes \\
\hline Aki et al. [31] & 2012 & 1 & Chest pain & INT. SEPT. & n.s. & Surg. & n.s. & Yes \\
\hline Beroukhim et al. [32] & 2012 & 1 & Palpitation & Pulm. Art. & n.s. & Surg. & n.s. & n.s. \\
\hline Imperatori et al. [23] & 2011 & 1 & HT. & INT. PERIC. & Yes & Surg. & No & No \\
\hline Al-Githmi et al. [33] & 2010 & 1 & Chest pain & Aortic root & n.s. & Surg. & n.s. & Yes \\
\hline Cong et al. [34] & 2011 & 1 & HT. & INT. PERIC. & Yes & Surg. & No & n.s. \\
\hline Ceresa et al. [35] & 2010 & 1 & HT. & L.A. & Yes & Surg. & No & Yes \\
\hline Petersen et al. [36] & 2010 & 1 & Palpitations & L.A. & Yes & Surg. & n.s. & Yes \\
\hline Gómez et al. [37] & 2010 & 1 & Resp. infect. & L.A. & n.s. & Surg. & No & No \\
\hline Rana et al. [38] & 2009 & 1 & HT. & INT. PERIC. & Yes & Surg. & n.s. & No \\
\hline Lorusso et al. [7] & 2009 & 1 & Chest pain & L.V. & No & Surg. & n.s. & Yes \\
\hline Zhou et al. [39] & 2009 & 1 & HT. & R.A.; R.V. & Yes & Surg. & No & Yes \\
\hline Tahir et al. [40] & 2009 & 1 & Chest pain & R.A. & No & Surg. & n.s. & Yes \\
\hline Thomas et al. [41] & 2009 & 1 & HT. & R.A. & Yes & Surg. & No & n.s. \\
\hline Alghamdi et al. [42] & 2009 & 1 & None & INT. PERIC. & n.s. & Surg. & No & n.s. \\
\hline Lee et al. [43] & 2009 & 1 & None & INT. PERIC. & n.s. & Surg. & n.s. & n.s. \\
\hline Brown et al. [6] & 2008 & 14 & 14/14 HT. & Heart & Yes & Surg. & $7 / 14$ & $2 / 14$ \\
\hline Vicente et al. [10] & 2008 & 1 & Wheezing & L.A. & No & Surg. & n.s. & No \\
\hline Hawari et al. [44] & 2008 & 1 & Chest pain & R.V. & No & Surg. & No & Yes \\
\hline Ali et al. [45] & 2007 & 1 & HT. & INT. PERIC. & n.s. & Surg. & n.s. & n.s. \\
\hline Maxey et al. [46] & 2007 & 1 & Palpitations & INT. SEPT. & n.s. & Surg. & n.s. & Yes \\
\hline Yuan et al. [47] & 2007 & 1 & HT. & R.A. & Yes & Surg. & No & n.s. \\
\hline Imren et al. [48] & 2007 & 1 & HT. & L.A. & n.s. & Surg. & n.s. & Yes \\
\hline Jimenez et al. [49] & 2005 & 1 & Palpitation & L.A. & n.s. & $\mathrm{CHT}$ & No & No \\
\hline Turley et al. [50] & 2005 & 1 & Chest pain & INT. SEPT. & No & Surg. & No & Yes \\
\hline Moorjani et al. [51] & 2004 & 1 & HT. & L.A. & Yes & Surg. & No & Yes \\
\hline Lupinski et al. [52] & 2004 & 1 & HT. & R.V. & Yes & Surg. & Yes & Yes \\
\hline Boumzebra et al. [53] & 2002 & 1 & None & INT. PERIC. & n.s. & Surg. & n.s. & Yes \\
\hline Tekin et al. [54] & 2000 & 1 & Dysphagia & L.A. & No & Surg. & n.s. & n.s. \\
\hline Pickering et al. [55] & 2000 & 1 & HT. & R.V. & Yes & Surg. & No & Yes \\
\hline Dresler et al. [56] & 1998 & 1 & HT. & L.A. & n.s. & Surg. & n.s. & Yes \\
\hline Hamilton et al. [5] & 1997 & 12 & HT. & 83\% L.A. & Yes & $\begin{array}{l}11 / 12 \\
\text { Surg. }\end{array}$ & n.s. & $2 / 11$ \\
\hline Cane et al. [57] & 2012 & 1 & n.s. & INT. SEPT. & n.s. & Surg. & n.s. & n.s. \\
\hline Casanova et al. [58] & 1996 & 1 & HT. & INT. PERIC. & Yes & Surg. & No & Yes \\
\hline Williams et al. [59] & 1994 & 1 & n.s. & INT. PERIC. & n.s. & Surg. & No & No \\
\hline Gomi et al. [60] & 1994 & 1 & HT. & L.A. & Yes & Surg. & No & Yes \\
\hline
\end{tabular}

Pts: patients; CPBP: cardiopulmonary bypass; Hered. Synd.: hereditary syndrome; Retroc.: retrocardiac; HT.: hypertension; n.s.: not specified; R.A.: right atrium; L.A.: left atrium; INT. SEPT.: interatrial septum; INT. PERIC.: intrapericardial; L.V.: left ventricular; R.V.: right ventricular; CHT: chemotherapy. 
was about $5 \mathrm{~cm}$. This suggests that the tumour mass grows undetected for long time before becoming symptomatic.

Little is known regarding long-term survival of IP patients, as long-term follow-up studies are not available.

The rarity of IP is well documented, only 30 cases being reported until 1992 [8]. We reviewed the medical literature subsequently published and found that over the two-decade period 1994-2013 three institutional series [5, 6, 9] including altogether 33 cases and 39 other individual case reports of IP were described (Table 1). In total, 102 cases of IP have been reported in the literature to date.

\section{Clinical and Diagnostic Features}

Arterial hypertension is the most common clinical presentation of IP; about $10 \%$ of reported paragangliomas are clinically silent (Table 1), the tumour being diagnosed incidentally after chest radiography, computed tomography (CT), or magnetic resonance (MR) imaging performed for unrelated reasons. More frequently the disease presents with paroxysmal symptoms of excess catecholamine production, which include hypertension, headache, palpitations, tremor, and facial pallor. Hypertension episodes are variable in frequency, severity, and duration and are difficult to treat. The hypertension crisis may induce arrhythmia and myocardial ischemia and may even be fatal. Bursts of catecholamine secretion can be provoked by a variety of events, which include accidental or surgical trauma, anaesthesia induction, invasive procedures, and eating food with high level of tyramine (e.g., red wine, chocolate, and cheese).

About 20\% of IPs are nonfunctioning (Table 1) and may present as a mass with symptoms related to compression of other organs, or they are incidentally discovered during imaging studies $[9,10]$.

The diagnosis of functioning paraganglioma requires biochemical testing to document elevated catecholamine secretion and is generally obtained by determining the plasma level of fractionated metanephrines during hypertensive crisis and the 24-hour urine normetanephrine level.

Imaging exams to localize the tumour and its metastases include CT and MR imaging, coupled with 123-Imetaiodobenzylguanidine (MIBG) scintigraphy. These exams provide anatomical and functional information with good sensitivity and specificity [11].

Some IP cases have been incidentally identified by chest radiography, as an enlargement of mediastinum or splaying of the carina by the tumour mass [5]. On CT scan IP typically appears as a well-enhancing mass, predominantly located in the posterior mediastinum or in the aortic-pulmonary window. The most frequent location is in close proximity to the left atrium (Table 1). CT and MR images allow to clarify tumour mass relationships with surrounding mediastinal structures and are of great importance for planning surgical resection.

\section{Treatment}

Surgical resection is the mainstay of treatment for benign and malignant IP. Resection must be completed with minimal tumour manipulation to prevent hypertensive crisis and tumour seeding. Control of tumour vascular supply requires adequate operative field exposure to avoid injury to surrounding organs. The operative access to the mediastinum depends on location and size of the lesion, as well as on the adjacent structures involved. Generally, the surgical approach to IP is with sternotomy, as cardiopulmonary bypass (CPBP) is usually planned for tumour removal. Less commonly, IP can be resected without CPBP, through left or right thoracotomy, depending on tumour location. If size and location of the lesion are favourable, the approach can also be with minimally invasive videothoracoscopic technique.

Patients with unresectable or metastatic disease may be treated with chemotherapy, radiofrequency thermoablation, cryoablation, and catecholamine blockade [11].

Perioperative management of IP patients is a challenge for anaesthesiologists and surgeons. Over the last 50 years, shared management of the patient involving also endocrinologists and cardiologists translated into a marked reduction of perioperative mortality from $40-60 \%$ to $0-6 \%$ [12].

\section{Perioperative Management of Hypertension}

In preparation for surgery, a detailed medical history is essential, along with physical examination, complete laboratory exams, and evaluation by cardiologist and anaesthesiologist. It is important to detect the presence of a cardiomyopathy or coronary artery disease by ECG, echocardiography, and coronary angiography, if necessary. However, for young patients without history of heart disease, it is debated whether preoperative ECG only is sufficient [13]. Echocardiography should be done to assess the presence of hypertrophic, dilated or Tako-Tsubo cardiomyopathy. This imaging technique is also useful to localize cardiac paraganglioma [14].

If hypertrophic cardiomyopathy as a result of chronic norepinephrine-induced hypertension is found, it is mostly symmetric and concentric $[12,13]$. Dilated cardiomyopathy has also been described.

An interesting finding in patients with functioning chromaffin tumours is the stress-related Tako-Tsubo cardiomyopathy, also called left ventricular apical ballooning syndrome. The pathophysiology of stress-induced and chromaffin tumour-induced cardiomyopathy is believed to be similar and mediated by catecholamines causing myocardial stunning [15]. Typically in Tako-Tsubo disease, the decreased ejection fraction caused by myocardial alterations undergoes spontaneous recovery. Common electrocardiographic findings in patients with catecholamine-secreting tumours are high QRS amplitudes with abnormal R, changes in STsegment and $\mathrm{T}$ waves, and prolongation of the Q-Tc interval [13].

Preoperative medical management to block the deleterious effects of excess catecholamine release and to allow plasma volume expansion is recommended [16]. Although $\alpha$-adrenoceptor antagonists, calcium-channel blockers, or angiotensin-receptor blockers have all been recommended, there are no evidence-based guidelines on the preferred 
drugs for preoperative catecholamine blockade. Consequently, there are widely ranging practices and international differences in perioperative pharmacologic management and approved therapies. Importantly, $\beta$-blockers should be used only after adequate pretreatment with $\alpha$-antagonists. Moreover, volemia expansion is also widely recommended before and after surgery [16].

5.1. Preoperative Optimization of Blood Pressure. Special preparation of the patient undergoing surgical removal of paraganglioma is necessary once clinical, instrumental, and laboratory evaluations are completed.

Without preoperative medical treatment, induction of anaesthesia or other stimuli can cause a hypertensive crisis, cardiac arrhythmias, and infarction or stroke, due to massive catecholamine release. Pharmacological treatment should be instituted for 1 to 2 weeks before surgery, to optimize cardiovascular function by relaxation of the constricted vasculature, expansion of the reduced plasma volume, and normalization of blood pressure. Normalization of blood volume reduces the risk of prolonged hypotension after tumour removal [16].

Preoperative antihypertensive therapy is useful in patients with sustained or paroxysmal hypertension. In this setting, interventions are focused on maintaining adequate plasma volume and on lowering blood pressure using $\alpha$ - and $\beta$-blockers. Alpha-adrenergic blockade should be started prior to $\beta$-adrenergic blockade to prevent acute hypertensive crisis. Alpha-blockade is generally initiated 7 to 10 days before surgery, using different drugs (phenoxybenzamine, prazosin, or doxazosin) to achieve a systolic blood pressure below $120 \mathrm{mmHg}$ when seated and below $90 \mathrm{mmHg}$ when standing. It is recommended that no blood pressure $>160 / 90 \mathrm{mmHg}$ values should be evident 24 hours before surgery, and an orthostatic hypotensive response (blood pressure $>80 / 45 \mathrm{mmHg}$ ) should be present. Moreover, no electrocardiographic ST/T segment changes at least for 1 week should be evident [17]. After reaching these blood pressure values, it is possible to initiate $\beta$-blocker administration, using low dosages to reduce the risk of negative inotropy [18]. Magnesium sulphate has also been shown useful to control blood pressure, when haemodynamic stability is difficult to achieve [18].

5.2. Intraoperative Management. During the operative period, it is essential to closely monitor cardiovascular function by electrocardiogram and to assess urine output, pulse oxinmetry, capnography, and body temperature. It may be necessary to monitor cerebral function with electroencephalography, if the patient has a recent history of cerebral infarction [19].

Cardiovascular monitoring requires an intra-arterial catheter and a central venous catheter to respond quickly to haemodynamic changes with vasoactive agents or fluid administration [13]. Pulmonary capillary wedge pressure monitoring may be useful because of discrepancy between right-sided and left-sided filling pressures, but the routine use of pulmonary catheters remains controversial. Transesophageal echocardiography is useful to optimise intravenous fluid administration and to assess perioperative cardiac function $[13,20]$.

5.3. Anaesthetic Technique. General anaesthesia is the most commonly chosen technique. Intravenous or inhalation agents have been used with success, but drugs that stimulate the sympathetic system responses or that may cause mechanical stimulation of the tumour by fasciculations, such as succinylcholine, should be avoided. Deep anaesthesia to inhibit cardiovascular response is more important than the specific agent used [13].

Several drugs are available to control blood pressure during tumour resection. Sodium nitroprusside should be used in patients with a history of acute myocardial infarction or congestive heart failure, although this drug has potential for overshoot hypotension [21]. Phentolamine is $\alpha$-adrenergic antagonist that can be given intravenously as continuous infusion or as boluses of 1 to $2 \mathrm{mg}$; it can cause tachycardia if the patient is not receiving $\beta$-blockers. Calcium channel blockers have some advantages over sodium nitroprusside, such as less risk of deep hypotension, no rebound hypertension, more controlled heart rate, and absence of cyanide toxicity [12]. Fenoldopam, a dopamine-1 receptor agonist that causes peripheral vasodilation and increases renal blood flow, may also be used.

After tumour removal, it is essential to control hypotension that may result from inadequate intravascular volume, residual $\alpha$-adrenergic antagonist effect, alteration of venous capacitance and haemorrhage. Priority should be given to volume replacement, which seems to be the main factor responsible for reduction of intraoperative mortality in chromaffin tumour surgery $[12,22]$. In addition to blood pressure control, it is necessary to carefully monitor blood glucose level, because hypoglycemia may develop after tumour removal, due to rebound hyperinsulinism, as the inhibitory effect of norepinephrine on insulin secretion is eliminated.

To illustrate the intraoperative management of anaesthesia, we report in Figure 1 the details of $\beta$-blocker and $\alpha$ adrenergic antagonist administration in a case undergoing surgery for removal of IP at our institution. The patient, a 34-year-old woman with a history of recent episodes of headache, palpitation, and flushing, had hypertension $(180 / 90 \mathrm{~mm} \mathrm{Hg})$ poorly controlled by ramipril. She was diagnosed with a functioning IP localized below the left atrial roof (Figure 2). Tumour resection was carried out through right thoracotomy without CPBP. A hypertensive crisis developed at the time of general anaesthesia induction $(210 / 100 \mathrm{~mm} \mathrm{Hg})$. During the critical steps of surgery, it was necessary to administer a greater amount of the anaesthetic drug sevoflurane, in association with multiple boluses of $\alpha$ - and $\beta$-blockers. Total labetalol boluses administered amounted to $125 \mathrm{mg}$, in addition to continuous infusion of labetalol $2 \mathrm{mg} / \mathrm{mL}$ (maximum administration of $0.266 \mathrm{mg} / \mathrm{min}$ ). Phentolamine was administered in boluses of $1-2 \mathrm{mg}$ (50 mg total) (Figure 1). 


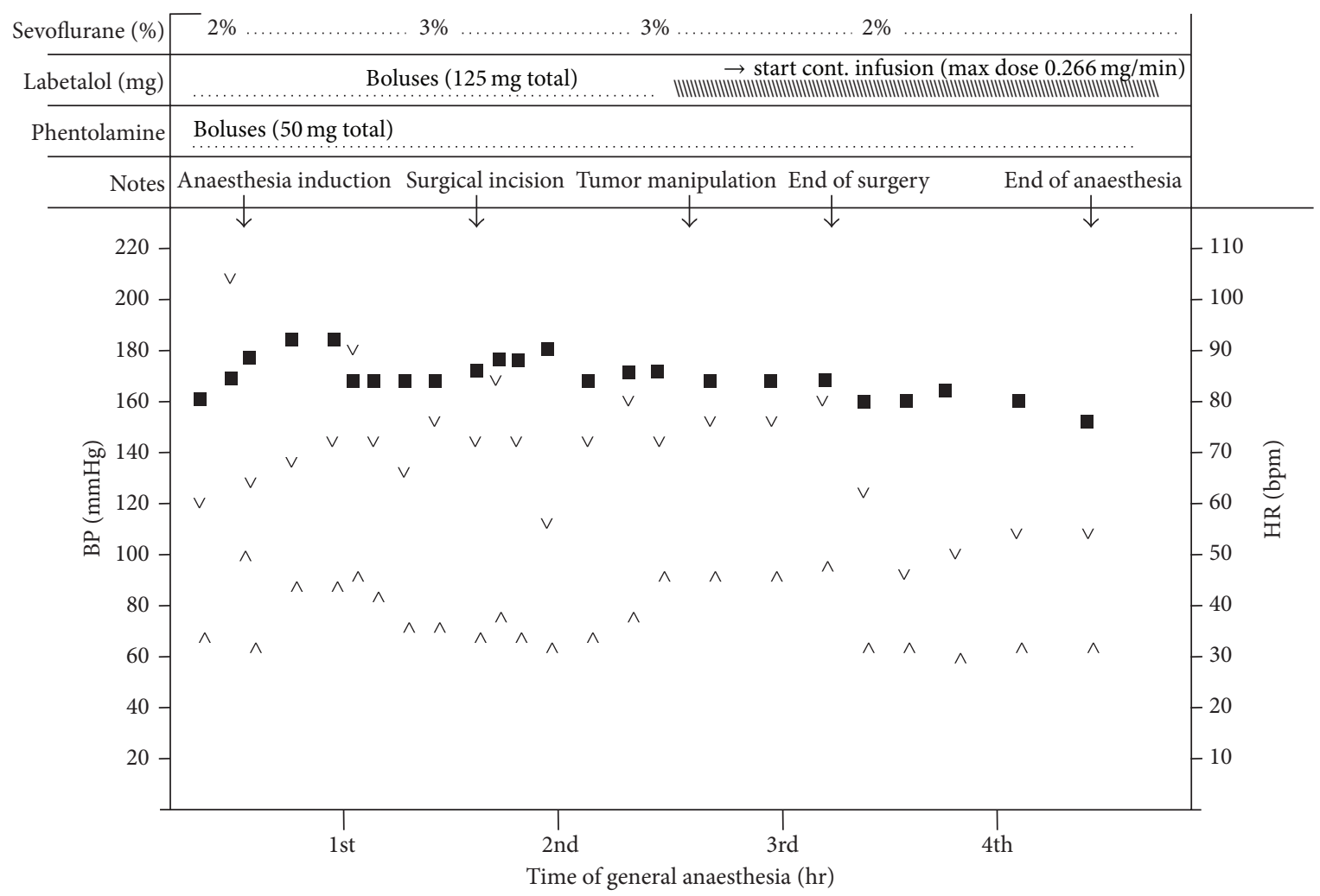

FIGURE 1: Anaesthesia management during surgery. $(\boldsymbol{\square})$ heart rate; $(\vee)$ systolic blood pressure; $(\wedge)$ diastolic blood pressure. As noted, during critical steps of surgery, it has been necessary to administer a greater amount of sevoflurane, in association with multiple boluses of beta-blocker. Total labetalol administered was $125 \mathrm{mg}+$ continuous infusion $2 \mathrm{mg} / \mathrm{mL}$ with maximum administration of $0.266 \mathrm{mg} / \mathrm{min}$. Phentolamine was administered by boluses of $1-2 \mathrm{mg}(50 \mathrm{mg}$ total).

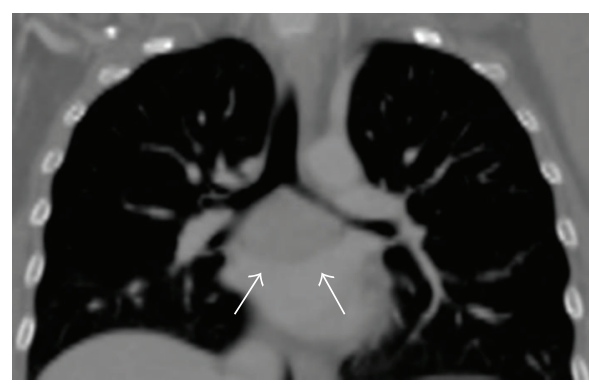

(a)

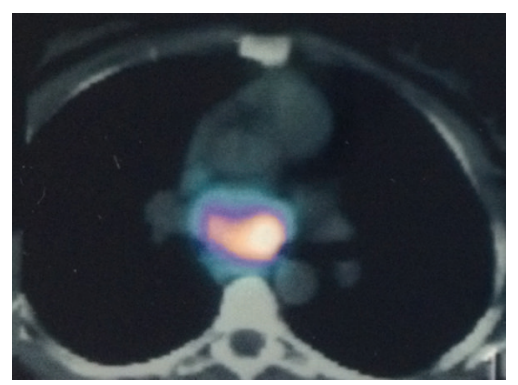

(b)

Figure 2: (a) Coronal view of chest computed tomography, revealing an intrapericardial $55 \mathrm{~mm}$ mass located at the roof of the left atrium (arrows). (b) ${ }^{123}$ I-metaiodobenzylguanidine chest scan showing high uptake by the intrapericardial mass.

After successful resection of the intrapericardial mass, which was histologically shown to be a chromogranin A-positive paraganglioma (Figure 3), the patient was discharged normotensive [23].

Some authors have questioned the validity of systematic preoperative optimization of blood pressure and volume expansion, drawing attention to the lack of evidence-based studies [22]. However, there is abundant literature to support a proper pharmacological preparation in all patients with functioning paraganglioma and pheochromocytoma, especially in subjects with cardiovascular risk.

We experienced that preoperative medical preparation of cardiac paraganglioma patients is critical. Alpha-adrenergic blockade (phenoxybenzamine or phentolamine) is usually started at the time of diagnosis and it is carried on preoperatively under close blood pressure monitoring, to prevent cardiovascular complications that may occur during surgery due to excess catecholamine secretion. These complications 


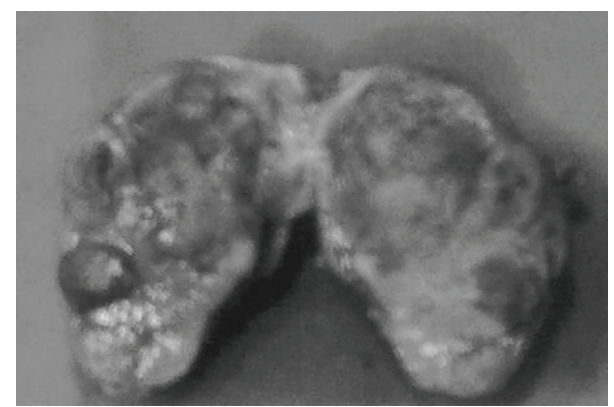

(a)

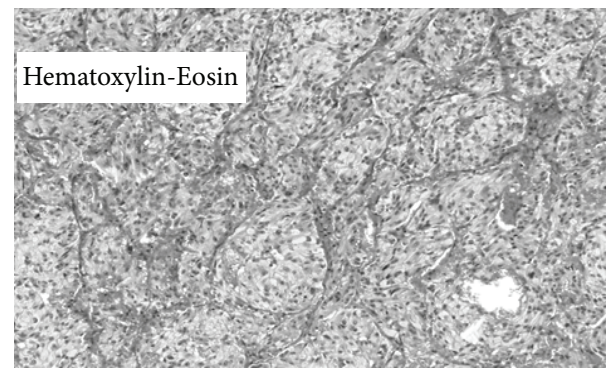

(b)

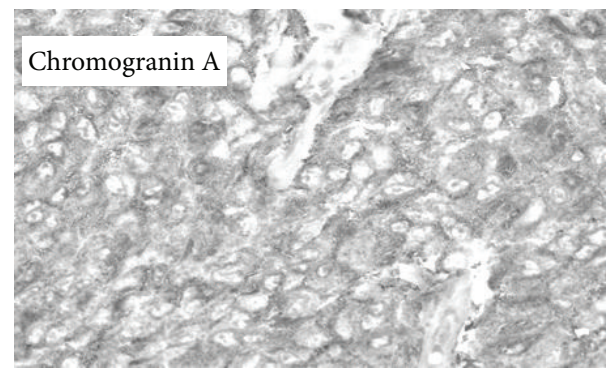

(c)

FIgURE 3: (a) Resected specimen $(55 \times 45 \mathrm{~mm}$ pseudocapsulated mass). Histology showed (b) paraganglioma with a nesting pattern (hematoxylin-eosin, $\times 200$ ) and (c) strong cytoplasmic immunoreactivity for chromogranin A $(\times 200)$.

include hypertensive crisis, arrhythmia, myocardial infarction, and pulmonary edema.

The goal of intraoperative pharmacologic therapy is to prevent sudden rise and fall of blood pressure. Betaadrenergic and calcium channel blockade can be used as adjuncts when blood pressure or tachycardia cannot be controlled [6].

At the time of tumour vascular supply control, rapid decrease of catecholamine level and rebound hypotension should be expected [24], and preoperative volume expansion helps in preventing it [6].

\section{Surgical Outcome and Follow-Up}

After surgical removal of IP, approximately $80 \%$ of patients become normotensive (Table 1). Persistent hypertension may be due to incomplete tumour resection or metastatic disease. The other $20 \%$ remain hypertensive without biochemical evidence of residual tumour, due to associated essential hypertension or to acquired renovascular changes [24].

Tumour recurrence in case of incomplete resection may occur; moreover, approximately 10\% IPs were found to be malignant; therefore, systematic follow-up is recommended. There is no general agreement on the method and frequency of follow-up after paraganglioma resection. Long-term follow-up is recommended with blood pressure measurements and periodic determination of urinary metanephrines; if necessary, 123-I-MIBG scintigraphy and CT imaging should be obtained [16].

\section{Conclusion}

IP is a rare tumour that may cause secondary hypertension. Surgical removal is the gold-standard treatment and normalizes blood pressure in about $80 \%$ of hypertensive cases; $20 \%$ remain hypertensive, likely due to associated essential hypertension. Preoperative medical preparation is critical to prevent cardiovascular complications that may result from excess catecholamine secretion during anaesthesia induction and surgical manipulation of the tumour. Alpha-adrenergic blockade and $\beta$-blockers are usually started at the time of diagnosis and this treatment is carried on perioperatively under close monitoring of blood pressure. Due to the rarity of functioning paraganglioma, systematic perioperative optimization of blood pressure and volume expansion has not been validated by randomized studies. However, there is abundant literature to support the value of pharmacological preparation and volume expansion to prevent cardiovascular complications.

\section{Conflict of Interests}

The authors declare that there is no conflict of interests regarding the publication of this paper.

\section{Acknowledgment}

The authors gratefully acknowledge Timothy Sampson for expert review of the paper.

\section{References}

[1] A. Moraitis and C. Stratakis, "Adrenocortical causes of hypertension," International Journal of Hypertension, vol. 8, pp. 1-10, 2011.

[2] P. A. Sarafidis and G. L. Bakris, "Resistant hypertension. An overview of evaluation and treatment," Journal of the American College of Cardiology, vol. 52, no. 22, pp. 1749-1757, 2008.

[3] R. A. DeLellis, R. V. Lloyd, P. U. Heitz et al., Pathology and Genetics of Tumours of Endocrine Organs, vol. 8 of WHO Classification of Tumour, IARC Press, Lyon, France, 2004.

[4] K. Y. Lee, Y.-W. Oh, H. J. Noh et al., "Extraadrenal paragangliomas of the body: imaging features," American Journal of Roentgenology, vol. 187, no. 2, pp. 492-504, 2006.

[5] B. H. Hamilton, I. R. Francis, B. H. Gross et al., "Intrapericardial paragangliomas (pheochromocytomas): imaging features," 
American Journal of Roentgenology, vol. 168, no. 1, pp. 109-113, 1997.

[6] M. L. Brown, G. E. Zayas, M. D. Abel, W. F. Young Jr., and H. V. Schaff, "Mediastinal paragangliomas: the mayo clinic experience," Annals of Thoracic Surgery, vol. 86, no. 3, pp. 946951, 2008.

[7] R. Lorusso, G. de Cicco, A. Tironi, S. Gelsomino, and R. de Geest, "Giant primary paraganglioma of the left ventricle," Journal of Thoracic and Cardiovascular Surgery, vol. 137, no. 2, pp. 499-500, 2009.

[8] V. A. Jebara, M. S. Uva, A. Farge et al., "Cardiac pheochromocytomas," Annals of Thoracic Surgery, vol. 53, no. 2, pp. 356-361, 1992.

[9] B. Ramlawi, E. A. David, M. P. Kim et al., "Contemporary surgical management of cardiac paragangliomas," Annals of Thoracic Surgery, vol. 93, no. 6, pp. 1972-1976, 2012.

[10] C. C. Vicente, E. Rodriguez, P. Aragoncillo et al., "Paracardiac nonfunctioning paraganglioma in a young woman," Circulation, vol. 118, no. 5, pp. 574-575, 2008.

[11] Pheochromocytoma and Paraganglioma Treatment, National Cancer Institute, http://www.cancer.gov/cancertopics/pdq/ treatment/pheochromocytoma/HealthProfessional.

[12] M. A. O. Kinney, B. J. Narr, and M. A. Warner, "Perioperative management of pheochromocytoma," Journal of Cardiothoracic and Vascular Anesthesia, vol. 16, no. 3, pp. 359-369, 2002.

[13] P. Van Braeckel, S. Carlier, P.-J. Steelant, L. Weyne, and L. Vanfleteren, "Perioperative management of phaeochromocytoma," Acta Anaesthesiologica Belgica, vol. 60, no. 1, pp. 55-66, 2009.

[14] M. F. James and L. Crojé, "Pheochromocytoma crisis: the use of magnesium sulfate," Anesthesia Analgiasia, vol. 99, pp. 680-686, 2009.

[15] K. E. Joynt, J. J. Moslehi, and K. L. Baughman, "Paragangliomas: etiology, presentation, and management," Cardiology in Review, vol. 17, no. 4, pp. 159-164, 2009.

[16] K. Pacak, "Approach to the patient: preoperative management of the pheochromocytoma patient," Journal of Clinical Endocrinology and Metabolism, vol. 92, no. 11, pp. 4069-4079, 2007.

[17] M. F. Roizen, B. D. Schreider, and S. Z. Hassan, "Anesthesia for patients with pheochromocytoma," Anesthesiology Clinics of North America, vol. 5, no. 2, pp. 269-275, 1987.

[18] J. M. Fox and P. H. Manninen, "The anaesthetic management of a patient with a phaeochromocytoma and acute stroke," Canadian Journal of Anaesthesia, vol. 38, no. 6, pp. 775-779, 1991.

[19] T. Ryan, A. Timoney, and A. J. Cunningham, "Use of transoesophageal echocardiography to manage beta-adrenoceptor block and assess left ventricular function in a patient with phaeochromocytoma," British Journal of Anaesthesia, vol. 70, no. 1, pp. 101-103, 1993.

[20] A. de La Chapelle, M. Deghmani, and B. Dureuil, "Peritoneal insufflation can be a critical moment during laparoscopic removal of phaeochromocytoma," Annales Francaises d'Anesthesie et de Reanimation, vol. 17, no. 9, pp. 1184-1185, 1998.

[21] J. M. Desmonts and J. Marty, "Anaesthetic management of patients with phaeochromocytoma," British Journal of Anaesthesia, vol. 56, no. 7, pp. 781-789, 1984.

[22] C. Lentschener, S. Gaujoux, A. Tesniere, and B. Dousset, "Point of controversy: perioperative care of patients undergoing pheochromocytoma removal-time for a reappraisal?" European Journal of Endocrinology, vol. 165, no. 3, pp. 365-373, 2011.
[23] A. Imperatori, L. De Monte, N. Rotolo et al., "Hypertension and intrapericardial paraganglioma: an exceptional presentation of multiple endocrine neoplasia type IIA syndrome," Hypertension, vol. 58, no. 5, pp. el89-e190, 2011.

[24] N. N. Nanna and D. E. Kenady, Pheochromocytoma. Surgical Treatment: Evidence-Based and Problem-Oriented, Edited by R. G. Holzheimer, J. A. Mannick, NCBI Bookshelf, 2001, http://www.ncbi.nlm.nih.gov/books/NBK7002.

[25] M. T. Gonzale-Lopez, S. G. Gonzalez, E. S. Garcia et al., "Surgical excision with left atrial reconstruction of primary functioning retrocardiac paraganglioma," Journal of Cardiothoracic Surgery, vol. 29, pp. 8-22, 2013.

[26] Q. X. Liu, H. Miao, C. Zhang et al., "Primary cardiac pheochromocytoma involving both right and left atria," Annals of Thoracic Surgery, vol. 95, pp. 337-340, 2013.

[27] Y. Wang, Y. Xiao, and X. Wang, "Primary cardiac paraganglioma arising from interatrial septum," Journal of Cardiothoracic Surgery, vol. 28, pp. 274-275, 2013.

[28] J. C. Tracy and R. O. Wein, "Intrapericardial paraganglioma associated with succinate dehydrogenase complex subunit $\mathrm{C}$ mutation syndrome," Head \& Neck, vol. 35, pp. 251-253, 2013.

[29] L. Marshall, P. Shah, S. Yeung, and J. Mundy, "Synchronous presentation of cardiac and abdominal paragangliomas," Annals of Thoracic Surgery, vol. 93, no. 5, pp. el15-e117, 2012.

[30] J. L. Huo, J. C. Choi, A. DeLuna et al., "Cardiac paraganglioma: diagnostic and surgical challenges," Journal of Cardiac Surgery, vol. 27, no. 2, pp. 178-182, 2012.

[31] A. Aki, K. Okada, H. Furuya et al., "Surgical treatment of an interatrial septal paraganglioma," Asian Cardiovascular and Thoracic Annals, vol. 20, pp. 581-583, 2012.

[32] R. S. Beroukhim, P. Del Nido, L. A. Teot, K. Janeway, and T. Geva, "Cardiac paraganglioma in an adolescent," Circulation, vol. 125, no. 5, pp. e322-e324, 2012.

[33] I. Al-Githmi, G. Baslaim, and N. Batawil, "Primary cardiac paraganglioma with dual coronary blood supply presenting with angina chest pain," Canadian Journal of Cardiology, vol. 26, no. 7, pp. e278-e279, 2010.

[34] L. Cong, M. Fan, W. Tian, M. Cheng, and G. Li, "Intrapericardial pheochromocytoma," Thoracic and Cardiovascular Surgeon, vol. 59, no. 3, pp. 179-181, 2011.

[35] F. C. Ceresa, F. Sansone, M. Rinaldi, and F. Patané, "Left atrial paraganglioma: diagnosis and surgical management," Interactive Cardiovascular and Thoracic Surgery, vol. 10, no. 6, pp. 1047-1048, 2010.

[36] J. Petersen, G. Cooper, P. Drew, B. Silverstein, and T. Beaver, "Paraganglioma resection requiring left atrial reconstruction," Clinical Cardiology, vol. 33, no. 3, pp. E75-E77, 2010.

[37] N. P. Gómez, G. M. Gómez, F. J. G. De Vargas, and C. P. Calvo, "Intrapericardial paraganglioma," Revista Espanola de Cardiologia, vol. 63, no. 1, pp. 116-117, 2010.

[38] O. Rana, P. Gonda, B. Addis, and K. Greaves, "Image in cardiovascular medicine. Intrapericardial paraganglioma presenting as chest pain," Circulation, vol. 119, no. 12, pp. e373-e375, 2009.

[39] J. Zhou, H.-T. Chen, J. Xiang, X.-H. Qu, Y.-Q. Zhou, and W.F. Zang, "Surgical treatment of cardiac pheochromocytoma: a case report," Annals of Thoracic Surgery, vol. 88, no. 1, pp. 278281, 2009.

[40] M. Tahir, S. J. Noor, A. Herle, and S. Downing, "Right atrial paraganglioma: a rare primary cardiac neoplasm as a cause of chest pain," Texas Heart Institute Journal, vol. 36, no. 6, pp. 594597, 2009. 
[41] D. Thomas, F. D. Grant, R. Kwong, V. Nose, M. F. Di Carli, and S. Dorbala, "Multimodality imaging of an unusual case of cardiac paraganglioma," Journal of Nuclear Cardiology, vol. 16, no. 4, pp. 644-647, 2009.

[42] A. A. Alghamdi, T. Sheth, Z. Manowski, O. F. Djoleto, and G. Bhatnagar, "Utility of cardiac CT and MRI for the diagnosis and preoperative assessment of cardiac paraganglioma," Journal of Cardiac Surgery, vol. 24, no. 6, pp. 700-701, 2009.

[43] C.-C. Lee, S. Chua, S.-C. Huang, F.-Y. Lee, and S.-Y. Chung, "Intrapericardial paraganglioma with intratumoral coronary arterial aneurysm and an arteriovenous fistula," Journal of the American Society of Echocardiography, vol. 22, no. 2, p. 211, 2009.

[44] M. Hawari, T. Yousri, R. Hawari, and G. Tsang, "Intrapericardial paraganglioma directly irrigated by the right coronary artery," Journal of Cardiac Surgery, vol. 23, no. 6, pp. 780-782, 2008.

[45] A. M. Ali, M. Devbhandari, A. Sastry, R. J. Ashleigh, and M. T. Jones, "Preoperative embolization followed by surgical excision of an intrapericardial pheochromocytoma," Annals of Thoracic Surgery, vol. 83, no. 1, pp. 302-304, 2007.

[46] T. S. Maxey, P. Grow, C. D. Morris, K. T. Patton, and R. A. Guyton, "Biatrial primary cardiac paraganglioma: a rare finding," Cardiovascular Pathology, vol. 16, no. 3, pp. 179-182, 2007.

[47] W.-Q. Yuan, W.-Q. Wang, T.-W. Su et al., "A primary right atrium paraganglioma in a 15-year-old patient," Endocrine, vol. 32, no. 2, pp. 245-248, 2007.

[48] Y. Imren, I. Tasoglu, A. A. Benson, and V. Sinci, "A rare intracardiac mass: cardiac paraganglioma," Heart Lung and Circulation, vol. 16, no. 2, pp. 116-117, 2007.

[49] J. F. Jimenez, E. T. Warren, R. K. Shroff, and G. A. Stolz, "Primary cardiac paraganglioma," The Journal of the Arkansas Medical Society, vol. 101, no. 12, pp. 362-364, 2005.

[50] A. J. Turley, S. Hunter, and M. J. Stewart, "A cardiac paraganglioma presenting with atypical chest pain," European Journal of Cardio-thoracic Surgery, vol. 28, no. 2, pp. 352-354, 2005.

[51] N. Moorjani, J. Kuo, and D. Wilkins, "Left atrial phaeochromocytoma," Heart, vol. 90, no. 11, p. e64, 2004.

[52] R. W. Lupinski, S. Shankar, T. Agasthian, C. H. Lim, and K. Mancer, "Primary cardiac paraganglioma," Annals of Thoracic Surgery, vol. 78, no. 3, pp. e43-e44, 2004.

[53] D. A. Boumzebra, Z. S. Charifchefchaouni, S. A. Belhadj, W. A. Maazouzi, and Z. Y. Al-Halees, "Intrapericardial paraganglioma," Saudi Medical Journal, vol. 23, no. 10, pp. 1278-1280, 2002.

[54] U. N. Tekin, I. A. Khan, N. Singh, V. M. Nair, B. C. Vasavada, and T. J. Sacchi, "A left atrial paraganglioma patient presenting with compressive dysphagia," Canadian Journal of Cardiology, vol. 16, no. 3, pp. 383-385, 2000.

[55] T. G. Pickering, O. W. Isom, G. W. Bergman, and J. M. Barbieri, "Pheochromocytoma of the heart," American Journal of Cardiology, vol. 86, no. 11, pp. 1288-1289, 2000.

[56] C. Dresler, J. Cremer, F. Logemann, and A. Haverich, "Intrapericardial pheochromocytoma," Thoracic and Cardiovascular Surgeon, vol. 46, no. 2, pp. 100-102, 1998.

[57] M. E. Cane, L. D. Berrizbeitia, S. S. Yang et al., "Paraganglioma of the interatrial septum," Journal of Cardiothoarcic Surgery, vol. 27, pp. 178-182, 2012.

[58] J. Casanova, C. S. Moura, J. P. Torres, L. Vouga, A. S. Graça, and M. M. R. Gomes, "Intrapericardial paraganglioma," European Journal of Cardio-thoracic Surgery, vol. 10, no. 4, pp. 287-289, 1996.
[59] K. S. Williams, B. K. Temeck, and H. I. Pass, "Intrapericardial pheochromocytoma complicated by massive intraoperative hemorrhage," Southern Medical Journal, vol. 87, no. 11, pp. 11641167, 1994.

[60] T. Gomi, T. Ikeda, J. Sakurai, Y. Toya, and M. Tani, "Cardiac pheochromocytoma. A case report and review of the literature," Japanese Heart Journal, vol. 35, no. 1, pp. 117-124, 1994. 


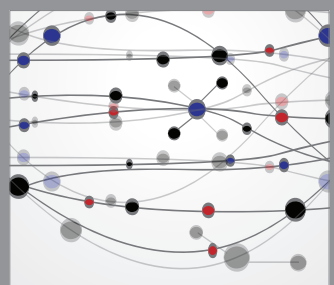

The Scientific World Journal
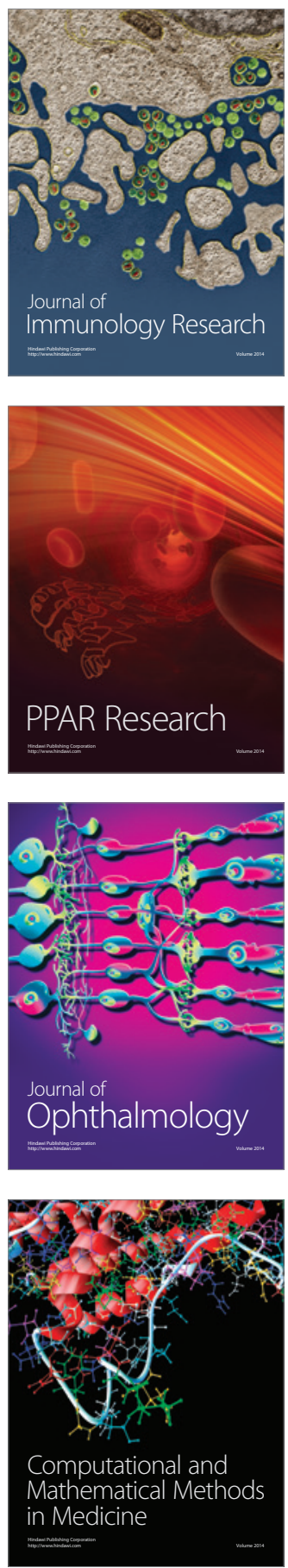

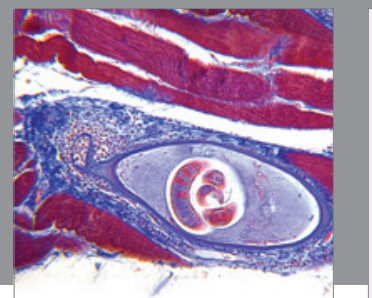

Gastroenterology

Research and Practice
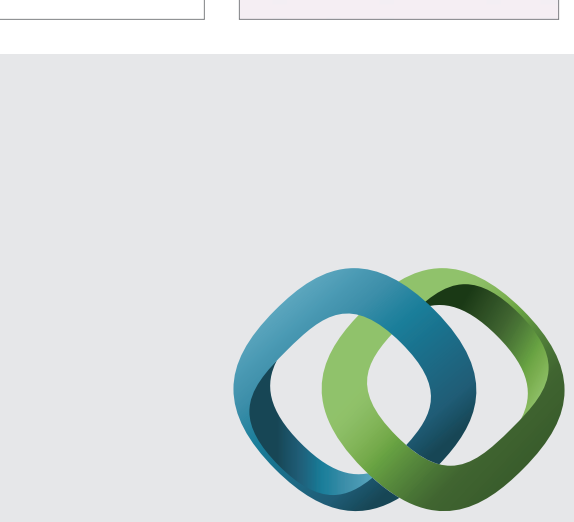

\section{Hindawi}

Submit your manuscripts at

http://www.hindawi.com
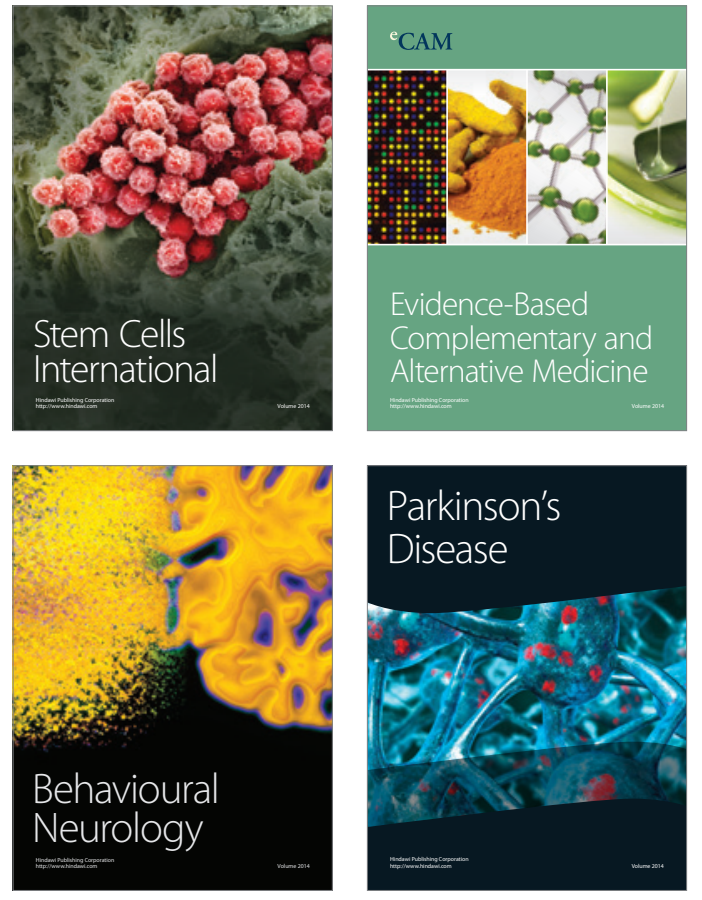
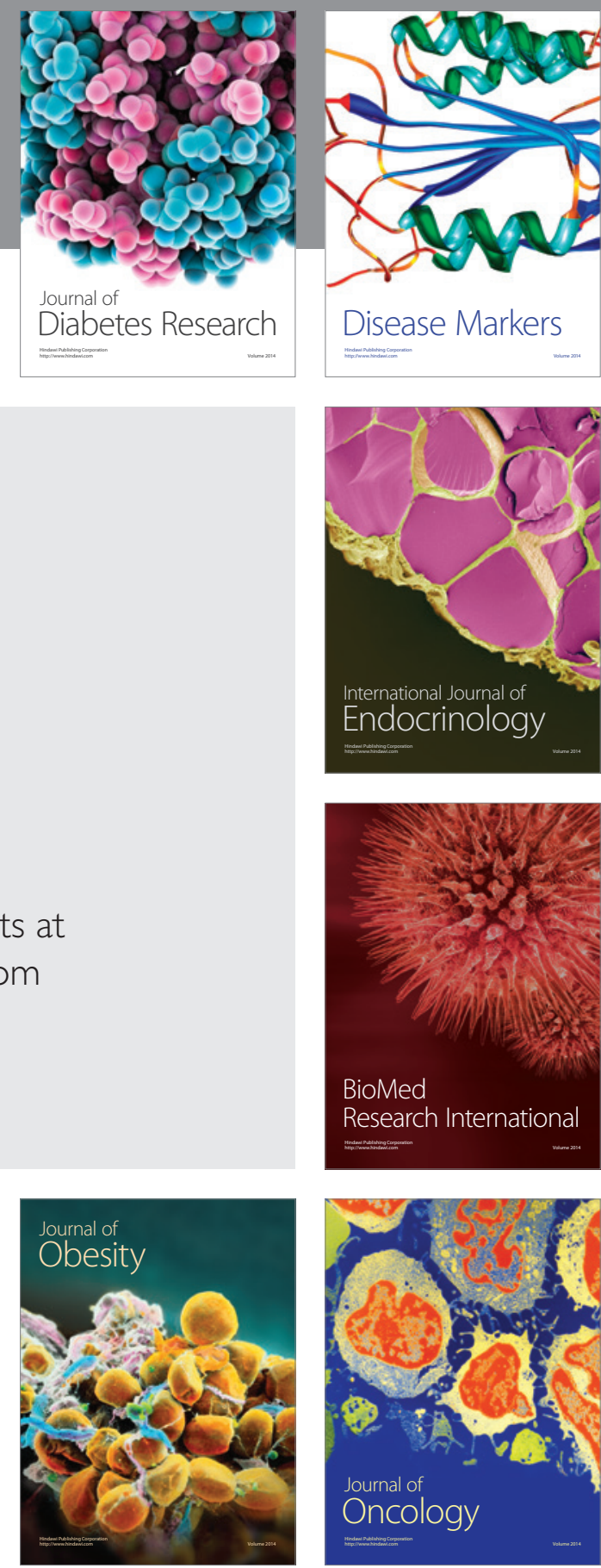

Disease Markers
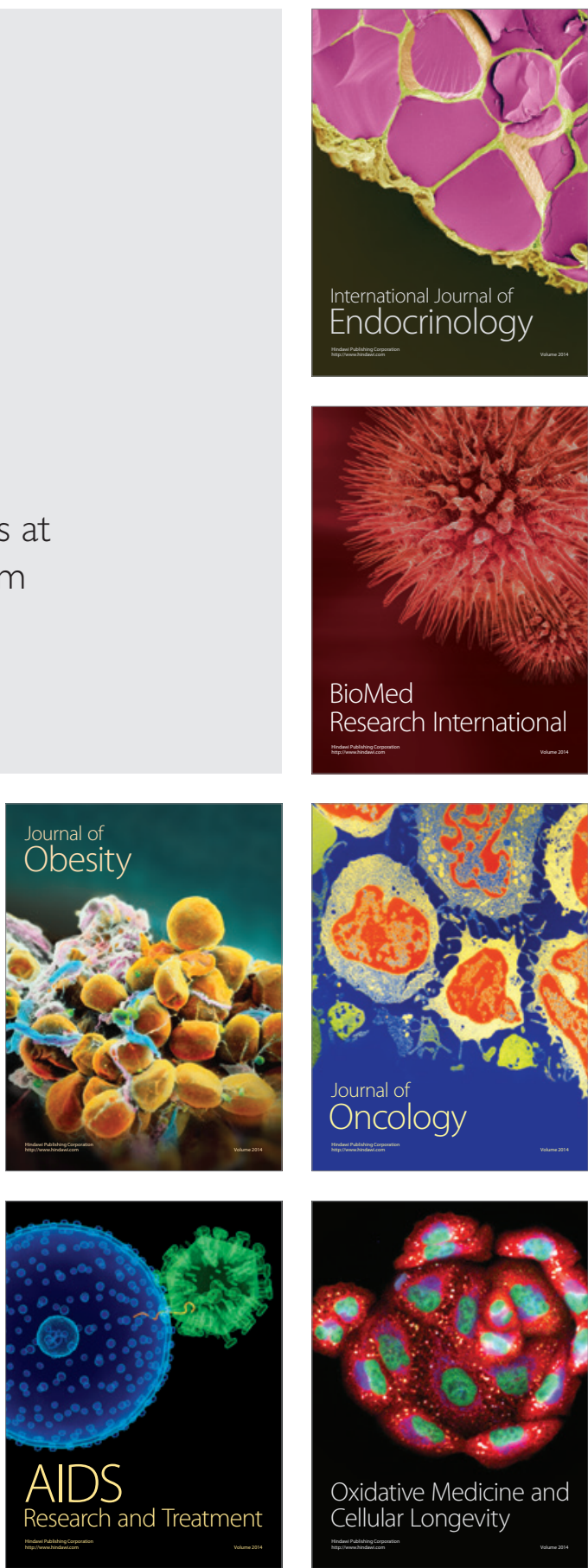